\title{
Philonsorbonne
}

12 | 2018

Année 2017-2018

\section{Agir dans l'angoisse ou par habitude ? La liberté de l'agent dans la philosophie existentialiste de Sartre}

Yoann MALINGE

\section{OpenEdition}

Journals

Édition électronique

URL : https://journals.openedition.org/philonsorbonne/1016

DOI : 10.4000/philonsorbonne.1016

ISSN : $2270-7336$

Éditeur

Publications de la Sorbonne

Édition imprimée

Date de publication : 1 janvier 2018

Pagination : 139-151

ISSN : 1255-183X

\section{Référence électronique}

Yoann MALINGE, « Agir dans l'angoisse ou par habitude ? La liberté de l'agent dans la philosophie existentialiste de Sartre », Philonsorbonne [En ligne], 12 | 2018, mis en ligne le 20 mars 2018, consulté le 08 juin 2021. URL : http://journals.openedition.org/philonsorbonne/1016 ; DOI : https://doi.org/ 10.4000/philonsorbonne.1016 


\title{
Agir dans l'angoisse ou par habitude ? La liberté de l'agent dans la philosophie existentialiste de Sartre
}

\author{
Yoann MALINGE \\ (Université Paris 1 Panthéon-Sorbonne) \\ (Centre de philosophie contemporaine)
}

La philosophie de Sartre dans L'Être et le néant est une philosophie existentielle. Il s'agit de montrer qu'exister, c'est toujours être en excès, être jeté là, avant même d'être tel ou tel, avant d'avoir une identité. Exister, c'est être-au-monde. Or être-au-monde, c'est précisément ne pas être du monde et donc être présent au monde. Pour cela, il faut comprendre l'être même de l'homme comme ouverture, comme éclatement - ce que permet la thèse de l'intentionnalité de la conscience, héritée de Husserl ${ }^{1}$ : la conscience est une visée, elle n'est pas ce qu'elle vise précisément parce qu'elle le vise, et elle n'est donc pas non plus une intériorité ${ }^{2}$. L'existence, ou plutôt l'ek-sistence, est dépassement de soi, dans un mouvement d'ouverture. Être jeté, c'est être sur le mode du changement et de la spontanéité ${ }^{3}$, par opposition au fait d'être englué, figé dans l'en-soi. Il est impossible dans ce travail de développer la dimension ontologique de la philosophie de Sartre. Cependant, c'est bien cette ontologie qui fonde les développements que Sartre consacre à l'angoisse et à la motivation. En effet, avant même de comprendre le Dasein heideggerien comme « réalité humaine ${ }^{4}$, Sartre a développé, dès $L a$ Nausée, une compréhension de l'existant comme être là, jeté sans raison

1. Voir notamment Husserl, Idées directrices pour une phénoménologie, §36 et $\$ 84$, trad. Paul Ricoeur, Paris, Gallimard, 2008. Voir aussi Sartre, «Une idée fondamentale de la phénoménologie de Husserl : l'intentionnalité », [1939], Situations I, Paris, Gallimard, 2010, p. $27-41$.

2. Sur ces développements trop rapides pour être précis et complets, voir La Transcendance de l'ego et l'article «Intentionnalité », op. cit.

3. Voir L'Être et le néant, Paris, Gallimard, 2007, p. 183.

4. Cette compréhension n'est pas extravagante. C'est ainsi que Corbin traduisit Dasein dans ce qui fut la première traduction en français de passages de Sein und Zeit. Voir le recueil des textes regroupés sous le titre Qu'est-ce que la métaphysique?, trad. Henry Corbin, Paris, Gallimard, 1938. 
dans le monde, sans nécessité 5 . Et c'est cette compréhension qui le conduit, dans L'Être et le néant, à envisager l'expérience de l'angoisse, expérience de l'indétermination. Selon Heidegger, l'homme est le lieu-tenant du néant ${ }^{6}$. Sartre va plus loin et affirme que l'homme est à lui-même " [s]on propre rien $\gg^{7}$. C'est cette thèse qui permet de comprendre l'angoisse dans la première partie de L'Être et le néant. Cette expérience est affirmée comme celle de la liberté. Or chez Sartre, la liberté, parce qu'elle est celle de l'existant n'est pas une faculté parmi d'autres, elle est le mode d'être de l'existant. L'existence est donc action.

Il semble ainsi pertinent d'envisager cette expérience de l'angoisse en une perspective de philosophie de l'action. Dès lors que la liberté n'est pas une faculté, que l'existant est compris comme néantisant, alors cette théorie de l'action ne peut pas reprendre le schéma aristotélicien qui divise le processus de l'action entre «savoir », « décider » et « vouloir ». L'angoisse semble au contraire être l'épreuve de ce qui sépare l'agent de ce qu'il connaît et de ce qu'il peut décider. Elle est l'expérience ontologique à partir de laquelle on doit comprendre l'agentivité comme ne relevant pas de la volonté mais d'un projet à la fois plus global et plus riche. Cependant, elle est aussi le nœud d'une difficulté : comment l'agent peut-il dépasser cette angoisse et agir? L'expérience de l'angoisse est donc un paradoxe : elle est à la fois l'expérience de la liberté, pourtant elle est aussi celle de l'inaction. À quoi bon être libre si cette liberté n'est pas mise en œuvre ? Si l'agent est séparé de ce qu'il est, s'il est également séparé des choses du monde par le néant qu'il est, comment l'agent peut-il se déterminer à agir ? Si l'on part du postulat que les actions humaines ont un sens, qu'elles s'inscrivent dans le monde pour en modifier la trame, sont-ce les choses du monde qui sont porteuses d'une valeur, d'un sens et qui deviennent par elles-mêmes les motifs de l'action? Ou bien, est-ce l'agent seul qui constitue les motifs de son action? L'expérience de l'angoisse répond à ce problème tout en reportant la difficulté de savoir comment le motif de l'action apparaît.

Pour répondre à ce problème, il convient alors d'étudier les deux formes d'angoisse, l'angoisse devant l'avenir et l'angoisse devant le passé. Cette analyse permettra alors de dresser les lignes directrices d'une théorie existentielle de la motivation.

\section{L'angoisse comme expérience de la liberté}

L'expérience de l'angoisse est l'épreuve de notre liberté. Lorsque l'existence a été définie comme néant, elle l'a d'abord été comme néant par

5. Voir La Nausée, in Sartre, CEuvres romanesques, Paris, Gallimard, Pléiade, 1981, p. 155.

6. Voir Heidegger, Questions I, Paris, Gallimard, Tel, 1990, p. 243.

7. L'Être et le néant, op. cit., p. 299.

8. Aristote, Éthique à Nicomaque II, 1105a30s. 
rapport à l'être de l'en-soi. Il faut maintenant aller plus loin en comprenant ce recul néantisant au sein de la conscience elle-même. En effet, ce n'est pas seulement du monde qu'elle est séparée, mais de ce qu'elle était auparavant.

Pour commencer, en reprenant l'analyse que Sartre emprunte à Kierkegaard $^{9}$, il faut distinguer l'angoisse de la peur ou crainte. La peur a un objet, peur d'une araignée, peur de la mort, autrement dit, elle est peur d'un être ou d'une situation du monde. L'angoisse, quant à elle, est «angoisse devant moi ${ }^{10}$, angoisse du néant que je suis. L'homme découvre que rien n'existe en lui qui le pousse à faire quoi que ce soit. Aucune entité (comme l'ego) n'intervient pour le déterminer. Il ne faut donc pas chercher une cause immanente préexistante comme déterminant de l'action ${ }^{11}$. À tel point que face à un précipice, il découvre que ce n'est pas tant la peur du vide qui le paralyse que l'angoisse devant son propre néant qui fait qu'il pourrait tout aussi bien sauter que ne pas sauter.

Pour bien comprendre cela, il faut rapporter la conscience à ce qu'elle peut faire, ce que Sartre appelle les «possibilités». Il faut distinguer les possibilités de ce que Sartre appelle des probabilités transcendantes. La différence ne tient pas au degré de réalisation mais au contenu et à la relation que l'agent entretient avec elles. Les possibilités sont des actions que l'agent pourrait faire. Par exemple, devant un précipice, le fait qu'une pierre tombe sur moi et me fasse tomber n'est pas une possibilité, c'est une probabilité. À l'inverse, que je me jette dans le précipice ou que je reste au bord sont bien des actions possibles. Ce sont des possibilités que peut viser la conscience. Ces possibilités possèdent-elles en elles-mêmes une force motrice? Constituent-elles des voies préalables que l'agent pourrait emprunter s'il le décidait?

Les réponses à ces questions doivent être négatives et le fondement se trouve dans l'être même du pour-soi. L'agent fait l'expérience de cette nature ontologique. Voilà pourquoi l'angoisse est l'expérience de la liberté. Dans l'angoisse, l'agent découvre qu'entre lui et ces possibilités existe un écart, un néant qui fait qu'aucune de ces possibilités ne comporte en ellemême une plus grande chance d'être réalisée. À l'instant où la conscience les vise, elle se rend compte qu'elle est séparée de ces deux possibilités par le même néant qui la tient en une position d'isolement. Ce qu'il importe ici de comprendre, c'est la thèse de la liberté comme isolement envers le monde et envers soi. L'angoisse est alors également l'expérience de la contingence de l'action, contingence qui est précisément celle de l'existence.

9. Kierkegaard, Le Concept de l'angoisse, I, 5, Paris, Gallimard, 1990, p. 201 sq. Sartre fait lui-même référence à Kierkegaard, notamment dans L'Être et le néant, op. cit., p. 64.

10. L'Être et le néant, op. cit., p. 64.

11. Voir les développements que Sartre consacre au caractère constitué de l'ego dans $L a$ Transcendance de l'ego. À ce sujet, je renvoie à Y. Malinge, «La Transcendance de l'ego », The Literary Encyclopedia. [https://www.litencyc.com/php/sworks.php?rec=true\&UID=20283] 


\section{L'angoisse devant l'avenir}

On peut maintenant envisager les deux types d'angoisse: l'angoisse devant l'avenir et l'angoisse devant le passé.

«Je m'angoisse parce que mes conduites ne sont que possibles et cela signifie justement que, tout en constituant un ensemble de motifs de repousser cette situation, je saisis au même moment ces motifs comme insuffisamment efficaces. Au moment même où je me saisis moi-même comme étant horreur du précipice, j'ai conscience de cette horreur comme non déterminante par rapport à ma conduite possible $»^{12}$.

La liberté se trouve justement dans cette absence de déterminisme de l'homme à agir de telle ou telle façon. Dans l'exemple du précipice, l'homme éprouve de l'horreur devant la situation qu'il affronte. N'est-ce pas là une émotion qui emporte des effets ? Dans son étymologie même, la passion indique que le sujet qui la vit subit une modification de son état premier. Or cette modification le conduit à rechercher ou à fuir l'objet qui a sur lui cet effet. L'horreur est cette passion que cause le vide et qui devrait conduire, selon cette logique, à fuir le vide en question. Pourtant, Sartre affirme que cette passion, cette horreur ne fait que présenter différentes possibilités qui, en elles-mêmes ne sont pas déterminantes. Pourquoi ?

La réponse ne réside pas dans un combat de la raison contre les passions. Á ce titre, il ne s'agit pas d'une lutte d'une faculté contre l'effet des événements extérieurs. Non pas que cette passion n'existe pas. Elle existe : c'est la peur. Et cette peur peut jouer un rôle dans l'action ${ }^{13}$. Ce qu'il importe de relever ici, c'est qu'il existe une angoisse en-deçà de cette peur. De cette angoisse, une théorie classique des passions ne peut rendre compte. Seules l'ontologie et la thèse de la conscience comme intentionnalité peuvent la fonder et l'expliquer.

En renonçant à penser l'agent comme un sujet, et la conscience comme une substance pensante, Sartre est conduit à revoir la relation de l'agent avec les possibilités de son action. En effet, quand l'agent se rapporte à ses possibilités, quand sa conscience les vise, il fait exister l'écart néantisant entre lui et elles. En les visant, il les tient à l'écart de lui puisque son être consiste justement en une puissance de néantisation. Même au moment où l'homme se saisit comme horreur du vide, il constate qu'il n'y a pas d'enpuissance dans cette horreur, qu'elle n'est pas en elle-même le moteur de son action. On peut donc déjà remarquer que le processus existentiel de l'action n'est pas un processus causal. Puisque la conscience n'est pas une substance pensante, puisqu'elle n'est pas une intériorité, il n'y a pas en elle quelque chose qui la pousse à agir. Au contraire, dès lors que la conscience vise quelque chose, elle s'en sépare.

12. L'Être et le Néant, op. cit., p. 66.

13. Voir L'Être et le Néant, op. cit., p. 66. 
Cependant, si on en reste là, on ne sait pas pourquoi la conscience choisit telle ou telle action. Il n'y a rien dans le monde ou en elle qui la pousse à agir. C'est justement lorsqu'elle appréhende son rapport avec les possibilités que naît l'angoisse.

«Cela veut dire qu'en constituant une certaine conduite comme possible et précisément parce qu'elle est mon possible, je me rends compte que rien ne peut m'obliger à tenir cette conduite $»^{14}$. «C $\mathrm{C}^{\prime}$ est précisément la conscience d'être son propre avenir sur le mode du n'être-pas que nous nommerons l'angoisse $»^{15}$.

Ces citations illustrent parfaitement la dimension existentielle de l'analyse de Sartre : en prenant conscience du néant, l'agent comprend que tous les possibles lui sont accessibles, et surtout qu'aucun n'a une plus grande probabilité d'exister. Il n'y a pas de certitude que demain il sera tel ou tel homme. Il n'y a pas plus de probabilité qu'il choisisse de sauter que de ne pas sauter. Rien ne le détermine. Les questions de degré de certitude (question subjective) et de degré de probabilité (question objective) ne sont plus pertinentes. Ce que décrit Sartre ici, c'est l'expérience du néant. Cette expérience se réalise en première personne, elle est ainsi doublement existentielle: d'une part, il s'agit d'une épreuve dans l'existence, une épreuve particulière, importante. D'autre part, c'est une expérience de l'existence et en cela, elle est cruciale : c'est par elle que le pour-soi prend conscience de son existence, qu'il en fait l'objet de sa visée et qu'il en découvre la teneur ontologique.

Pourtant ne pourrait-on pas penser qu'il y a une continuité entre l'être actuel de l'agent et l'être qu'il sera le lendemain s'il fait telle action ? Il y a certes une continuité ${ }^{16}$, mais elle ne saurait suffire à entraîner l'action de l'agent. En effet, ce que nous envisageons ici, c'est bien un mouvement de l'agent pour réaliser quelque chose. Or quand l'agent se réfère à ce qu'il pourrait faire, à l'être qu'il pourrait être s'il accomplissait telle action, il s'y réfère sur le mode négatif de ce qu'il n'a pas encore fait, de ce qu'il n'est pas encore. Ce «n'être-pas» n'est pas seulement une négation formelle et extérieure. Sartre a bien montré qu'une telle négation est interne et qu'elle trouve son origine dans le néant qu'est le pour-soi ${ }^{17}$. L'angoisse est ce rapport particulier que l'agent entretient avec son avenir.

Ce n'est pas le moi présent qui peut entraîner l'agent à agir puisqu'il se tient à distance de ce qu'il n'est pas encore. On ne peut pas parler de l'agent comme d'un substrat, parce que le «je suis » actuel n'est pas le fondement

14. L'Être et le Néant, op. cit., p. 66.

15. L'Être et le Néant, op. cit., p. 67.

16. Il n'est pas possible de traiter ici la question de la temporalité. Sur ce point, voir $L$ ' $\hat{E}$ tre et le néant, deuxième partie, chapitre 2.

17. Le chapitre premier de la première partie de L'Être et le néant a pour titre « l'origine de la négation ». 
de ce que je serai. On voit donc bien le souci sartrien de déconstruire le sujet et toute idée de permanence sous-jacente. En effet, avec une pensée de la conscience comme néantisante, l'être que je serai, est un objet que vise la conscience et qu'elle n'est donc pas.

\section{L'angoisse devant le passé}

Une autre angoisse existe, celle devant mon passé affirme Sartre. Quand le joueur, qui a pris la résolution de ne plus jouer, voit une table de jeu,

«ce qu'il saisit alors, c'est précisément la totale inefficience de la résolution passée. Elle est là, sans doute, mais figée, inefficace, dépassée du fait même que j'ai conscience d'elle. Elle est moi encore, dans la mesure où je réalise perpétuellement mon identité avec moi-même à travers le flux temporel, mais elle n'est plus moi du fait qu'elle est pour ma conscience. Je lui échappe, elle manque à la mission que je lui avais donnée. Là encore, je la suis sur le mode du n'être-pas. Ce que le joueur saisit à cet instant, c'est encore la rupture permanente du déterminisme, c'est le néant qui le sépare de lui-même : j'aurais tant souhaité ne plus jouer ${ }^{18}$.

Deux thèses sont déjà affirmées ici. Tout d'abord, une thèse concernant la temporalité : l'absence d'efficience du passé. Cette négation d'une force du passé trouve elle aussi une origine ontologique, celle du néant qu'est l'agent et celle de la théorie intentionnelle de la conscience. En effet, en tant que la résolution passée est pour la conscience, elle est donc son objet et la conscience n'est donc pas cette résolution passée. En refusant de faire de la conscience une intériorité et de l'agent un sujet, on isole l'agent de son passé. Si la spontanéité est le mode d'être de la conscience, alors elle ne peut pas être contrainte par un vécu de conscience antérieur. C'est donc bien la critique d'une conception de la conscience comme intériorité substantielle qui trouve ici ses effets.

En outre, cette expérience de l'angoisse devant le passé renvoie à l'impossibilité de faire de soi un être, c'est-à-dire de l'en-soi. L'agent ne peut pas être un joueur repenti. Il ne sera jamais celui qu'il est, il est séparé de son Ego par un néant. La thèse qu'a développé Sartre dans $L a$ Transcendance de l'Ego trouve ici son application concrète. Si l'Ego est second, s'il est constitué, il ne peut pas être l'auteur des conduites. L'Ego est un être constitué et transcendant, il n'a pas d'efficience, il ne peut pas intervenir dans le vécu de conscience de l'agent. Ce que l'agent est, il l'est sur le mode de «ne l'être pas », et il a donc un rapport néantisant à ses décisions.

18. L'Être et le Néant, op. cit., p. 68. 
On pourrait alors objecter qu'il peut se reconnaître dans ses décisions passées, qu'il y a entre elles et lui un rapport de ressemblance. Cependant, l'objection tombe d'elle-même. Dès lors qu'il y a une reconnaissance possible dans la décision passée, dès lors qu'existe un rapport entre elle et l'agent, existe alors également une distance et une séparation. Ce moi passé qui a pris la décision de ne plus jouer, ce n'est pas moi, actuellement. Le rapport de reconnaissance contient synthétiquement deux instances qu'il s'agit de rapprocher, l'une qui reconnaît et l'autre qui est reconnue. Mais dès lors qu'il y a reconnaissance, il y a division. Même si la reconnaissance est un rapport de proximité, ce n'est pas une fusion. L'agent qui se reconnaît dans une décision passée est donc bien séparé d'elle par un néant. Même si l'agent avait voulu faire de sa résolution quelque chose d'efficace, il ne saurait se lier lui-même, être cause de lui-même à partir d'une résolution passée. Là encore, il faut souligner le fait que la motivation ne peut pas prendre la forme d'une causalité.

\section{Le problème de l'habitude}

Dans cette expérience de l'angoisse devant le passé, Sartre envisage le passé récent et constitué d'un seul acte, celui du serment. Peut-être que ce serment a été mûri mais il ne constitue qu'un événement dans la pratique et dans la durée de la vie du joueur. À l'inverse, le jeu constitue pour lui une «habitude», bien que Sartre n'utilise pas ce concept. Il faut essayer de reprendre l'analyse de l'exemple afin de comprendre les raisons de ce silence sur le passé antérieur au serment, celui de l'habitude de jouer. Cela éclairera la conceptualisation sartrienne de l'action.

D'une part, l'habitude est réitération d'une même activité. D'autre part, et surtout, cette réitération produit une facilitation dans l'accomplissement de l'acte. Cette facilitation trouve sa condition de possibilité dans le fait qu'il reste quelque chose après chaque effectuation de l'acte. Refaire un même acte engendre des effets sur l'agent, en plus des effets de l'action dans le monde. En effet, l'habitude n'est pas seulement la succession d'une même action, c'est aussi, selon Félix Ravaisson dans De l'habitude, la formation d'une «disposition », d'une «vertu ${ }^{19}$, une «nature acquise, une seconde nature ${ }^{20}$. L'habitude permet une forme de spontanéité qui n'est pas seulement la mise en œuvre d'un réflexe, mais aussi une meilleure adaptation à la situation. Cependant, si l'action devient «plus libre et plus prompte » en devenant «davantage une tendance, un penchant», souvent alors elle «se dérobe entièrement et sans retour à la volonté et à la conscience ${ }^{21}$ affirme Ravaisson.

19. Ravaisson, De l'habitude, Paris, Rivages poche, 1996, p. 30.

20. De l'habitude, op. cit., p. 83.

21. De l'habitude, op. cit., p. 70. 
Est-ce à dire que « ça agit » en nous ?

«La loi de l'habitude ne s'explique que par le développement d'une spontanéité passive et active tout à la fois, et également différente de la fatalité mécanique, et de la liberté réflexive $»^{22}$.

Pour Ravaisson, l'habitude n'est ni le développement d'un principe sur lequel l'agent n'a pas de contrôle, ni non plus la réalisation volontaire d'un désir. L'habitude est intermédiaire, sans s'opposer à la volonté. En effet, la tendance s'inscrit dans la continuité de la volonté, elle poursuit les mêmes fins que la volonté lorsque celle-ci était nécessaire à l'initiation de l'action. Selon Ravaisson, l'habitude modifie la relation que l'agent entretient avec la fin qu'il souhaite atteindre par son mouvement. Le mouvement n'est plus le moyen d'atteindre une fin qui est à distance de l'agent. À mesure que l'habitude se développe, la fin devient le mouvement lui-même, "la fin se confond avec le mouvement $»^{23}$. L'idéal se réalise dans ce mouvement. «L'habitude est de plus en plus une idée substantielle », souligne Ravaisson. Par exemple, pour l'habitude de jouer, on peut considérer que si le joueur a commencé à jouer c'était pour atteindre une fin précise qui existe en dehors du jeu : disons gagner de l'argent. Jouer était le moyen de gagner de l'argent. Mais avec l'habitude, la fin réside dans le mouvement lui-même, si bien que l'on peut dire que le joueur joue pour jouer.

On peut maintenant essayer de comprendre pourquoi Sartre n'envisage pas le concept d'habitude. Le problème se pose sur deux plans: d'une part, sur les conséquences qu'une pensée de l'habitude emporte pour la compréhension de la conscience. D'autre part, sur la relation qu'entretient l'agent avec son action habituelle.

Il faut commencer par mettre au jour les conditions qui permettent l'existence de l'habitude. S'il reste quelque chose comme une disposition qui se développe par la réitération d'un même acte, c'est qu'il existe sous cette disposition, une nature pour la recevoir. Quand, après Ravaisson, nous avons qualifié l'habitude de "seconde nature », cela implique logiquement qu'il y ait une première nature. Soit c'est cette première nature qui est le support de la seconde, soit elle est remplacée par la seconde parce qu'il existe un support neutre pour la recevoir. Dès lors, on peut requalifier le serment du joueur de ne plus jouer. Dans l'exemple de Sartre, la décision est comprise isolément, sans tenir compte de l'habitude. Mais si on conceptualise le passé du joueur comme habitude, alors la décision de ne plus jouer n'est plus qu'un instant dans la durée et dans la répétition de l'activité habituelle. Sartre néglige ce point qui participe pourtant de la reprise du jeu. Il est vrai que la résolution reste séparée de l'agent par le néant intraconscientiel mais pourquoi le joueur reprend-il le jeu ? Dans une analyse qui ferait droit à l'habitude, la reprise du jeu s'explique par le fait que l'activité de jouer

22. De l'habitude, op. cit., p. 76.

23. De l'habitude, op. cit., p. 78. 
consiste en la réitération d'une activité déjà pratiquée, réitération qui ne demande pas d'effort puisque l'activité en question se trouve facilitée par l'habitude. Elle ne fait pas appel à la décision volontaire, à la réflexion, elle s'inscrit dans une volonté générale qui n'est pas réfléchie.

Sartre ne dit rien de cette habitude parce qu'il ne veut rien donner à la tendance. En pensant la conscience comme la structure qui consiste à se jeter vers l'objet, Sartre fait de la conscience une activité. Il ne lui est donc pas possible de penser le caractère passif de l'habitude, et a fortiori la passivité de l'addiction.

Pour autant, en logique sartrienne, on pourrait faire droit à la transcendance des motifs qui consiste à reprendre le jeu. L'agent n'est-il pas séparé des motifs de jouer par le même néant qui le sépare des motifs de ne pas jouer? Ce néant en tant qu'il est le mode d'être de la conscience isole l'agent de tout motif. Rien ne le détermine à choisir ce motif plutôt qu'un autre. On retrouve ici les analyses de l'angoisse devant l'avenir.

Toutefois, cette angoisse ne serait-elle pas estompée voire annulée par la facilité que présente le fait de suivre son habitude ? Autrement dit, le motif de suivre son habitude n'est-il pas doté d'une valeur particulière en raison même de la facilité qu'elle engendre, le jeu étant alors l'objet qui viendrait combler le manque du pour-soi ? Dès lors, ce serait l'agent lui-même qui aurait fixé une certaine valeur à ce motif. Ne serait-il pas alors prisonnier de cette valeur? Peut-il sortir du projet qui valorise les possibles de l'action habituelle ? Ne s'est-il pas enfermé lui-même, se conduisant à faire de cette décision de ne plus jouer un faux engagement dans un projet qu'il n'a pas réellement adopté ? Pour Sartre, il semble que l'habitude ne soit pensée que comme recours de l'homme de mauvaise foi, mais ne serait-ce pas le serment qui aurait été pris de mauvaise foi ?

On le voit, le concept d'habitude conduit à une aporie dans la relation de l'agent à son action. Le changement d'habitude, comme le changement de projet est laissé dans l'ombre. Que le joueur ne tienne pas son engagement de ne plus jouer, on peut le comprendre avec la théorie du néant qu'est le pour-soi, mais qu'il continue de jouer reste un mystère si l'on ne fait pas droit à l'habitude. Ou bien, il faut essayer de dépasser cette conceptualisation de l'habitude en utilisant la conceptualisation sartrienne du projet. Mais demeure la question du changement possible du projet. On voit avec l'exemple du joueur qu'il ne suffit pas de vouloir changer de projet pour y parvenir. C'est un point important de la théorie sartrienne de la motivation. La volonté n'est pas une faculté de l'esprit qui commanderait l'action. Si elle peut intervenir, et surtout si elle veut être efficace, la volonté doit s'inscrire dans un projet existentiellement vécu. Sans une telle inscription, la délibération volontaire est inefficace ${ }^{24}$, elle demeure une apparence que l'agent met en œuvre pour dissimuler un engagement réel qui n'est pas

24. Le serment est l'expression d'une volonté. Or, la liberté ne repose pas sur la volonté. Sur ce point, voir l'article d'André Barata « Towards a theory of action in Sartre's philosophy. From action to ethics », Philonsorbonne, 12 | 2018, p. 125-126. 
assumé comme tel. Autrement dit, dans l'exemple du joueur, la délibération qui a conduit l'agent à prendre volontairement la décision de ne plus jouer, est inefficace parce qu'elle demeure ponctuelle, elle ne constitue pas un engagement au sens fort du terme. S'engager, ce n'est pas seulement prendre telle ou telle décision, c'est être-au-monde d'une certaine manière. En faisant de l'engagement une dimension ontologique qui concerne l'être-aumonde de chaque homme, la modification possible de cet engagement exige autre chose qu'une délibération et l'exercice de la volonté. Puisqu'elle concerne l'être-au-monde de l'homme, peut-être faudrait-il alors une véritable révolution subjective pour en changer ${ }^{25}$.

D'autre part, l'habitude emporte une modification importante dans l'ontologie même de l'action. Si elle conduit à faire du mouvement la fin visée, et si, en elle «se confondent le réel et l'idéal » ${ }^{26}$, alors l'action trouve sa raison d'être dans le mouvement même qui la constitue. Autrement dit, l'habitude ne conduit-elle pas à négliger un caractère essentiel de l'action qui est de modifier le monde selon une intention?

Sur ce point, la pensée de Sartre parvient à s'extraire de la difficulté en distinguant les gestes des actions. Cette distinction est développée dans Saint Genet, comédien et martyr: le geste n'est qu'un phénomène qui permet d'atteindre, par sa répétition, l'être de la persona. En latin, persona désigne «les masques portés à la scène par les acteurs tragiques ou comiques ». «Par métonymie, persona désignait également le rôle joué par l'acteur portant le masque ${ }^{27}$. Le geste est alors l'avènement de cette persona. Il consiste à faire apparaître ce qui est déjà là, à agir selon un être déjà présent. Ce faisant, on peut dire que le geste opère sur son opérateur une forme d'aliénation, il est l'expression d'une forme d'assujettissement à soi-même, comme si l'action était conditionnée par l'être de l'agent. Ce faisant, le geste fait disparaître le néant qu'est la conscience. Il est à la fois une constitution et une manifestation de cette persona, l'être substantiel du «comédien » $\mathrm{Genet}^{28}$. On voit donc que l'on retrouve les deux éléments constituants de l'habitude : la réitération d'un même mouvement et la nature créée par cette répétition. À l'inverse, l'action n'est pas l'effet de cet être substantiel.

«La liberté c'est l'être humain mettant son passé hors de jeu en sécrétant son propre néant. (...) C'est continuellement que la conscience se vit ellemême comme néantisation de son être passé $»^{29}$.

25. Dans l'œuvre de Sartre, ce point est problématique et nous essayons de le traiter au cours de nos recherches doctorales. Pour un premier aperçu, par distinction avec la psychanalyse freudienne, voir Juliette Simont, «Le choix originel: destin et liberté » in Les Temps modernes, $\mathrm{n}^{\circ}$ 674-675, juillet-octobre 2013, p. 68-93.

26. De l'habitude, op. cit., p. 79.

27. Paul Clavier, Dictionnaire culturel en langue française, tome III, Alain Rey (éd.), Paris, Le Robert, 2005, p. 1589.

28. Saint Genet, comédien et martyr, Paris, Gallimard, 2011, p. 75.

29. L'Être et le Néant, op. cit., p. 63. 
La liberté ne réside pas dans la volonté ou la délibération. Être libre, c'est être néantisant de ce qui est son propre passé et mais aussi son avenir. Cette puissance de néantisation isole l'agent dans ses décisions et ses actions. Elle lui permet d'agir et non pas seulement de faire des gestes. La liberté consiste précisément dans cet isolement qui confère à l'agent une responsabilité totale de ce qu'il fait. Il est le seul principe de son action.

\section{L'angoisse et la liberté d'indifférence}

Si la théorie sartrienne de l'action est fondée sur une ontologie et sur une théorie de la conscience comme intentionnalité et spontanéité, alors la conscience semble isolée du monde et d'elle-même, isolée des motifs qui serait à l'origine de l'action. Dès lors, il s'agit de savoir ce qui distingue l'expérience de l'angoisse devant l'avenir de la liberté d'indifférence?

L'angoisse devant l'avenir est l'appréhension du néant qui sépare l'agent des différentes actions possibles qui s'offrent à lui. Une telle description de l'angoisse semble rappeler la description de la liberté de l'âne de Buridan qui devant un seau d'avoine et un seau d'eau n'est pas plus incliné à choisir l'un que l'autre. Cette liberté que l'on peut qualifier, à la suite de Descartes, de liberté d'indifférence n'est-elle pas le plus bas degré de la liberté ${ }^{30}$ ? Chez Descartes, la liberté d'indifférence est la liberté qui ignore ce qu'elle veut, faute de savoir ce qui est vrai et ce qui est bien. On peut donc penser alors que l'angoisse devant l'avenir relève de cette liberté d'indifférence, incapable de savoir ce qui est bien pour elle.

Il faut se garder d'aller trop loin dans cette mise en parallèle. D'abord, Sartre n'affirme pas que l'angoisse est la liberté, mais seulement la conscience de la liberté. Ainsi, lorsque l'agent éprouve l'angoisse devant lui-même, et devant l'absence de détermination à sauter plutôt que de ne pas sauter, il prend seulement conscience de l'existence de sa liberté. Mais cette liberté ne réside pas dans cette angoisse, elle réside dans le fait que l'homme peut accorder une valeur différente aux différents motifs qui l'entraînent à choisir une action plutôt qu'une autre. Cette valorisation des motifs dépend du projet originel. La liberté chez Sartre ne saurait donc être réduite à cette liberté d'indifférence, puisqu'elle réside précisément dans le choix d'un projet. Même si l'adoption du projet est problématique, il est possible d'envisager le rapport entre le projet et les motifs. La valeur des motifs dépend du projet, comme être-au-monde. Toutefois, cette dépendance n'empêche pas l'agent de revoir cette valorisation, soit ponctuellement pour tel ou tel motif, soit globalement pour changer de projet.

30. Voir Descartes, Méditations métaphysiques, IV et « Lettre à Mesland du 9 février 1645 » «l'indifférence me semble signifier proprement l'état dans lequel se trouve la volonté lorsqu'elle n'est pas poussée d'un côté plutôt que de l'autre par la perception du vrai ou du bien ; et c'est en ce sens que je l'ai prise lorsque j'ai écrit que le plus bas degré de la liberté est celui où nous nous déterminons aux choses pour lesquelles nous sommes indifférents ». 
$\mathrm{Au}$ terme de cette étude de l'angoisse, il faut marquer encore deux points.

Le premier concerne la temporalité de l'action et son déroulement. La conscience est nue et spontanée, elle vit dans un présent qui l'isole. En affirmant que le joueur se trouve obligé de faire vivre à nouveau toutes les raisons qui l'ont conduit la veille à prendre la décision de ne plus jouer, Sartre montre que la conscience ne peut pas construire volontairement un Moi. Pour être suivies d'effets, les décisions doivent être prises au présent. Il s'agit à nouveau pour Sartre de rejeter un processus causal de l'action. Les décisions prises hier ne peuvent être la cause des actions d'aujourd'hui. Il faut que l'intention et l'action soient simultanées. On peut même aller plus loin : toute intention qui n'est pas réalisée aussitôt par une action n'est pas une véritable intention ${ }^{31}$. Tant qu'on en reste au stade de la pensée des motifs, il n'y a qu'une pure visée. L'intention doit donc être comprise en un sens restreint, en un sens pratique. Avoir une véritable intention, c'est déjà commencer à agir. Ainsi, la décision prise par le joueur doit être déclassée : ce n'est pas une intention. Par là, il s'agit aussi de développer corrélativement une critique morale de la «bonne intention ». Il n'y a pas de «bonne intention» qui existe sans que l'action bonne existe. Il n'y a donc pas d'excuse. Ce faisant, l'homme est donc responsable de lui-même, il ne tient qu'à lui d'agir selon son projet et sa valorisation, de faire de cette valorisation des motifs une intention au sens fort du terme. Sans quoi, se retrancher derrière de bonnes intentions qui ne seraient pas suivies d'action, c'est se draper dans une «belle âme », c'est se retirer du monde et se consoler dans le petit intérieur douillet de la bonne conscience.

Le second point concerne le problème de la liberté. Sartre en une prolepse répond à une objection possible, mais qui n'est pas anodine. Une objection d'inspiration spinoziste: l'angoisse n'est-elle pas ignorance des causes réelles de mon action? Si l'agent éprouve de l'angoisse devant l'action à réaliser, n'est-ce pas parce qu'il ignore ce qui le pousse à agir ? En cela, il n'y aurait pas de liberté. Néanmoins, il ne faut pas se méprendre sur le rôle que joue l'angoisse. Elle n'a pas un rôle de preuve de notre liberté. La preuve de cette liberté réside dans le fondement ontologique mis au jour par la thèse du néant. C'est parce que l'être de l'homme est néant, capacité à interroger et donc à s'extraire de l'en-soi pour faire surgir en lui la possibilité

31. Cf. L'Être et le Néant, op. cit., p. 529 : « Notre description de la liberté, ne distinguant pas entre le choisir et le faire, nous oblige à renoncer du coup à la distinction entre l'intention et l'acte. On ne saurait pas plus séparer l'intention de l'acte que la pensée du langage qui l'exprime et, comme il arrive que notre parole nous apprend notre pensée, ainsi nos actes nous apprennent nos intentions, c'est-à-dire nous permettent de les dégager, de les schématiser, et d'en faire des objets au lieu de nous borner à les vivre, c'est-à-dire en prendre une conscience non-thétique ». Il ne s'agit pas de nier l'existence des intentions non effectives, il s'agit de les penser comme ayant un autre statut en raison de leur absence de portée matérielle. Tant qu'elle reste de l'ordre de l'idée, elle est une façon de viser le monde, mais elle n'est pas ellemême l'objet de la visée intentionnelle. Cela ne signifie pas qu'elle est inconsciente. Elle est plutôt une conscience non positionnelle. 
du non-être qu'il est libre. L'angoisse n'est que la conscience de la liberté, conscience que les motifs ne suffisent pas en eux-mêmes à faire agir et que c'est l'agent qui leur donne de la valeur. Sartre procède donc à une réduction du pouvoir des motifs. Ceux-ci n'ont que le pouvoir que l'agent leur donne. La liberté n'est donc pas une résistance à un déterminisme, elle n'existe qu'à la condition de bien reconnaître l'absence de pouvoir des motifs en euxmêmes. Ainsi, ce n'est pas une liberté d'indifférence que Sartre décrit. Ce qu'il montre, c'est l'incapacité des motifs en eux-mêmes à faire agir. Par conséquent, il accorde un pouvoir important à l'agent, puisque c'est lui qui est l'auteur des motifs, il en est donc responsable ${ }^{32}$.

Par conséquent, si l'angoisse révèle une liberté vertigineuse, celle-ci concerne particulièrement l'action ponctuelle qui prend place dans un projet originel déjà déterminé. En revanche, si l'agent croit pouvoir, par une décision volontaire, modifier radicalement le cours de sa vie, il s'aperçoit que sa décision exige d'être vécue, c'est-à-dire d'être reprise en un engagement. Il apparaît donc que le projet originel n'est pas soumis au choix volontaire d'une liberté absolue. Le cours d'une vie ne relève pas d'une liberté de l'ordre du caprice. Pour autant, le passé ne détermine pas l'avenir. C'est donc sur une ligne de crête qu'il faut placer le projet originel, en tant que l'individu en est responsable mais qu'il ne peut le modifier sans un engagement réel, ce qui accroît donc sa responsabilité ${ }^{33}$.

32. Il nous restera à montrer ailleurs comment la présence des autres dans la situation peut venir perturber cette création des motifs de l'action et l'engagement de l'agent dans son projet originel.

33. J'ai pu présenter une première version de ce travail dans le cadre du projet de recherche «Rationalité pratique, jugement et motivation», soutenu par le programme PHC Pessoa (30848UF), associant l'université Paris 1 Panthéon-Sorbonne et l'Universidade da Beira Interior. Je tiens à remercier Laurent Jaffro et José Manuel Santos de m'y avoir associé. 